\title{
Heteroclinic intersections between Invariant Circles of Volume-Preserving Maps
}

\author{
H. E. Lomelí \\ Department of Mathematics \\ Instituto Tecnológico Autónomo de México \\ México DF 01000 \\ J. D. Meiss ${ }^{\dagger}$ \\ Department of Applied Mathematics \\ University of Colorado \\ Boulder CO 80309-0526
}

November 18, 2018

\begin{abstract}
We develop a Melnikov method for volume-preserving maps with codimension one invariant manifolds. The Melnikov function is shown to be related to the flux of the perturbation through the unperturbed invariant surface. As an example, we compute the Melnikov function for a perturbation of a three-dimensional map that has a heteroclinic connection between a pair of invariant circles. The intersection curves of the manifolds are shown to undergo bifurcations in homology.
\end{abstract}

\section{AMS classification scheme numbers:}

34C20,34C35,34C37,58F05,70H99

\section{Introduction}

Volume-preserving maps on $\mathbb{R}^{3}$ provide an interesting and nontrivial class of dynamical systems and give perhaps the simplest, natural generalization to higher dimensions of the

${ }^{*}$ HL wants to thank the Math department at UCSC for their hospitality during the academic year 2000-2001. Useful conversations with R. Montgomery are gratefully acknowledged. Also he would like to thank the support of Asociación Mexicana de Cultura A.C.

†Support from NSF grant DMS-0202032 gratefully acknowledged 
much-studied class of area-preserving maps. They also arise in a number of applications such as the study of the motion of Lagrangian tracers in incompressible fluids or of the structure of magnetic field lines [1, 2]. Experimental methods have only recently been developed that allow the visualization of particle trajectories in three-dimensional fluids [3, 4. The infinite dimensional group of volume-preserving diffeomorphisms is also at the core of the ambitious program to reformulate hydrodynamics $[5]$.

While some of the results for area-preserving maps generalize to the volume-preserving case, the study of transport in such systems is still in its infancy [6, 0, 8]. The theory of transport is based on dividing phase space into regions separated by partial barriers through which flux can be measured. For the area-preserving case, the natural partial barriers are formed from the stable and unstable manifolds of periodic orbits or cantori [9, 10]. Primary intersections can be used to form resonance zones [11, 12]-regions of phase space that are bounded by alternating stable and unstable segments joined at primary intersection points. Because the intersection points are primary, a resonance zone is bounded by a Jordan curve and has an exit and an entry set [13]. The area of each of these sets is the geometric flux, the area leaving the resonance zone each iteration of the map. The images of the exit and entry sets and their intersections completely define the transport properties of the resonance zone [14].

Thus the beginning of a generalization of this theory to higher dimensions is the study the intersections of codimension-one stable and unstable manifolds for volume-preserving maps.

As is well-known, a transversal intersection of stable and unstable manifolds is associated with the onset of chaos, giving rise to the construction of Smale horseshoes. A widely used technique for detecting such intersections is the Melnikov method. Given a system with a pair of saddles, and a heteroclinic or saddle connection between them, the Melnikov function computes rate at which of change the distance between the manifolds changes with a perturbation. The integral of the Melnikov function between two neighboring primary intersection points is the first order term in the geometric flux [15, 16].

Most applications of the method are for two-dimensional maps and flows 117, 18, 19, 20], though a Melnikov method for a three-dimensional incompressible flow was developed in [21]. In this latter case the perturbation may be periodically time dependent, and the Poincaré map of the system is assumed to have a hyperbolic invariant curve, with twodimensional manifolds.

For the case of maps, the analogue of Melnikov integral is an infinite sum whose domain is the unperturbed connection. As usual, a simple zero of this function corresponds to a transverse intersection of the manifolds for the perturbed map. We developed a Melnikov method for three-dimensional maps in [22] to study and classify intersections of stable and unstable manifolds for fixed points.

In this paper we generalize this method to the problem of detecting heteroclinic orbits between a pair of normally hyperbolic invariant sets in volume-preserving maps on $\mathbb{R}^{n}$. Our application is to the case of invariant circles for a three-dimensional map.

To obtain a Melnikov function, we must define an appropriate measure of the distance between a manifold and its perturbation. In [22] we used the cross product of pair of 
tangent vectors fields to obtain this distance. Different versions of Melnikov's method have used other ways of measuring the splitting between the unperturbed separatrix and the perturbed one, though naturally only the normal distance is well-defined in the codimension-one case. This is appropriately measured using an adapted normal vector field or differential form. An adapted normal is a normal field to the saddle connection that is invariant under the dynamics. If the map is integrable, then the gradient of an integral can be used to construct the adapted normal, but the concept applies more generally to nonintegrable systems.

We use the Melnikov function to construct a flux-form, an ( $n-1)$-form whose integral over a fundamental domain on the connection measures the first order flux through the connection. The fundamental domain is an annulus that generates the entire manifold upon iteration. Since the map is volume-preserving, the net (algebraic) flux always vanishes, but the one-way (geometric) flux gives a measure of the transport.

In 22] we introduced a family of volume-preserving maps that have a saddle connection between a pair of fixed points. This family is obtained from a family of planar twist maps with a saddle connection [23]. This family can be modified so that it has a pair of invariant circles with a corresponding of saddle connection. We perturb this family by composing it with a near-identity, volume-preserving map, thus producing examples of volume-preserving maps with transverse heteroclinic orbits.

We study the curves of zeros of the Melnikov function on a fundamental domain of the unperturbed manifold. Using the map to identify the two boundaries, the fundamental domain becomes a torus. Thus the zeros of the Melnikov function can be characterized by their homology on this torus. We show that as the parameters of the map are varied, the homology of these curves undergoes bifurcations, and that these bifurcations strongly influence the geometric flux.

\section{Basic definitions and properties}

Suppose $f_{0}: \mathbb{R}^{n} \rightarrow \mathbb{R}^{n}$ is a diffeomorphism on $n$-dimensional Euclidean space. A smooth perturbation of $f_{0}$ is a family of functions $f_{\varepsilon} \equiv f(\cdot, \varepsilon)$ such that $f(\cdot, 0)=f_{0}$ and $f(x, \varepsilon)$ is smooth in both variables. We now define a vector field on $\mathbb{R}^{n}$ that will be used to measure the motion of an invariant manifold.

Definition (Perturbation vector field). Given a perturbation $f_{\varepsilon}$ of $f_{0}$, define the vector field $X_{\varepsilon}$ for any point $x \in \mathbb{R}^{n}$ by

$$
X_{\varepsilon}(x) \equiv\left[\frac{\partial}{\partial \varepsilon} f_{\varepsilon}(y)\right]_{y=f_{\varepsilon}^{-1}(x)} .
$$

Perturbation vector fields have some special properties. First, it is easy to see that $X_{\varepsilon}$ is independent of $f_{0}$. Second, if one regards $X_{\varepsilon}$ as a time dependent vector field (where time is $\varepsilon)$, then $y(\varepsilon)=f_{\varepsilon}(x)$ is the solution of the initial value problem

$$
\frac{d y}{d \varepsilon} \equiv X_{\varepsilon}(y), \quad y(0)=f_{0}(x) .
$$


Thus if we let $F_{t, s}=f_{t} \circ f_{s}^{-1}$, then $F$ represents the flow of the nonautonomous vector field $X_{\varepsilon}$ [24, Thm. 2.2.23]. Since $F$ is volume-preserving, the vector field $X_{\varepsilon}$ has zero divergence with respect to $\Omega$ [24, Thm. 2.2.24].

It is often convenient to define a perturbed family by composing $f_{0}$ with an $\varepsilon$ dependent perturbation:

$$
f_{\varepsilon}=\left(i d+\varepsilon P_{\varepsilon}\right) \circ f_{0}=f_{0}+\varepsilon P_{\varepsilon} \circ f_{0} .
$$

In this case, the vector field is the first order approximation to the perturbation, $X_{0}(x)=$ $P_{0}(x)$.

\subsection{Invariant manifolds}

Suppose the family $f_{\varepsilon}$ has a family of invariant manifolds $\mathcal{W}_{\varepsilon} \hookrightarrow \mathbb{R}^{n}$. In this paper, we will assume that $\mathcal{W}_{\varepsilon}$ is a codimension-one surface. Our goal is to understand, at least to first order, the relation between the perturbation vector field and the way these invariant manifolds evolve with $\varepsilon$. Later, we will restrict ourselves to the case in which $\mathcal{W}_{\varepsilon}$ consists of pieces of stable and unstable manifolds of some invariant set. When $\mathcal{W}_{\varepsilon}$ is a smooth graph over $\mathcal{W}_{0}$, we can define a map that is adapted to the $\varepsilon$ parameterization:

Definition (Adapted deformation). A map $\phi: \mathcal{W}_{0} \times\left(-\varepsilon_{0}, \varepsilon_{0}\right) \rightarrow \mathbb{R}^{n}$, is adapted to $\mathcal{W}_{\varepsilon}$, if there is an $\varepsilon_{0}>0$ such that

- $\phi_{\varepsilon}=\phi(\cdot, \varepsilon)$ is a diffeomorphism $\phi_{\varepsilon}: \mathcal{W}_{0} \rightarrow \mathcal{W}_{\varepsilon}, \forall \varepsilon \in\left(-\varepsilon_{0}, \varepsilon_{0}\right)$.

- $\phi_{0}=\phi(\cdot, 0)=i d_{\mathcal{W}_{0}}$.

There is quite a bit of freedom in the choice of $\phi$; however, only the normal behavior is important for our application, since it measures the actual motion of $\mathcal{W}_{\varepsilon}$ with $\varepsilon$, and this is unique:

Proposition 1. Suppose $\phi_{\varepsilon}$ and $\tilde{\phi}_{\varepsilon}$ are two adapted deformations for a family of invariant manifolds $\mathcal{W}_{\varepsilon}$. Then

$$
\left[\frac{\partial}{\partial \varepsilon} \tilde{\phi}_{\varepsilon}(x)-\frac{\partial}{\partial \varepsilon} \phi_{\varepsilon}(x)\right]_{\varepsilon=0} \in T_{x} \mathcal{W}_{0} .
$$

Proof. Since both $\phi_{\varepsilon}(x)$ and $\tilde{\phi}_{\varepsilon}(x)$ are points in $\mathcal{W}_{\varepsilon}$, the curve $C_{\varepsilon}(x)=\phi_{\varepsilon}^{-1}\left(\tilde{\phi}_{\varepsilon}(x)\right)$ is a curve in $\mathcal{W}_{0}$ parameterized by $\varepsilon$, and $C_{0}(x)=x$. Thus its derivative at zero is a tangent vector to $\mathcal{W}_{0}$ :

$$
\left[\frac{\partial}{\partial \varepsilon} C_{\varepsilon}\right]_{\varepsilon=0} \in T_{x} \mathcal{W}_{0}
$$

Thus

$$
\left.\frac{\partial}{\partial \varepsilon} \phi_{\varepsilon}\left(C_{\varepsilon}(x)\right)\right|_{\varepsilon=0}=\left.\frac{\partial}{\partial \varepsilon} \phi_{\varepsilon}(x)\right|_{\varepsilon=0}+D \phi_{0}(x)\left[\frac{\partial}{\partial \varepsilon} C_{\varepsilon}(x)\right]_{\varepsilon=0}=\left.\frac{\partial}{\partial \varepsilon} \tilde{\phi}_{\varepsilon}(x)\right|_{\varepsilon=0},
$$

Since $D \phi_{0}(x)=I$, this gives the promised result. 
We will use this proposition to compute the Melnikov function in $\S$. For this we need to measure rate of change of an invariant manifold with respect to a perturbationchanges in the tangent direction are unimportant. In order to measure the change in the normal direction, we introduce the concepts of adapted normal vector fields and adapted forms.

\section{$2.2 \quad$ Adapted normals}

We will measure the splitting by using a normal to the invariant manifold $\mathcal{W}_{0}$, that throughout this paper will be a codimension-one submanifold, i.e. a surface. To be useful, the normal field should evolve in a precise way under the unperturbed map or, as we say, be "adapted" to the dynamics.

First we recall some notation. Let $v$ be a vector field and $f$ a diffeomorphism. The pull-back of $v$ under $f$ is $\left(f^{*} v\right)(x)=(D f(x))^{-1} v(f(x))$. Similarly, the pull-back of a $k$-form $\omega$ is $\left(f^{*} \omega\right)_{x}\left(v_{1}, v_{2}, \ldots v_{k}\right)=\omega_{f(x)}\left(D f(x) v_{1}, \ldots, D f(x) v_{k}\right)$. Finally the inner product of a vector field $Y$ with a $k$-form $\omega$ is defined as the $(k-1)$-form $i_{Y} \omega=\omega(Y, \cdot, \ldots, \cdot)$.

Definition (Adapted normal field). Suppose that $f: \mathbb{R}^{n} \rightarrow \mathbb{R}^{n}$ is a diffeomorphism with an invariant surface $\mathcal{W}$, and there is given an inner product $\langle$,$\rangle for vectors on \mathbb{R}^{n}$. An adapted normal field is a smooth function $\eta: \mathcal{W} \rightarrow \mathbb{R}^{n}$ such that

- $\eta(x) \neq 0$ for all $x \in \mathcal{W}$.

- $\eta(x)$ is normal to the surface for all $x \in \mathcal{W}$, that is $\eta(x) \in T_{x} \mathcal{W}^{\perp}$.

- For all vector fields $Y: \mathcal{W} \rightarrow \mathbb{R}^{n}$ we have that

$$
f^{*}\langle\eta, Y\rangle=\left\langle\eta, f^{*} Y\right\rangle .
$$

The geometry is shown in Fig. 1. Note that the pullback of a scalar function $g: \mathbb{R}^{n} \rightarrow \mathbb{R}$ is $f^{*} g(x)=g(f(x))$; thus if we define $Z(x)=f^{*} Y(x)$, (3) is equivalent to

$$
\langle\eta(x), Z(x)\rangle=\langle\eta(f(x)), D f(x) Z(x)\rangle
$$

Adapted normals can be thought of as a generalization of the gradient normal that one gets from a first integral. Recall that the gradient of a smooth function $J: \mathbb{R}^{n} \rightarrow \mathbb{R}$ is the unique vector field, $\nabla J$, such that for all vector fields $Y$ on $\mathbb{R}^{n}$,

$$
i_{Y} d J \equiv d J(Y)=\langle\nabla J, Y\rangle .
$$

If $f$ has a nondegenerate first integral, $J=J \circ f$, then (4) implies that $f^{*}\langle\nabla J, Y\rangle=$ $\left\langle\nabla(J \circ f), f^{*} Y\right\rangle$. Therefore, if the diffeomorphism $f$ has a first integral $J$, then $\nabla J$ is an adapted vector field, provided it doesn't vanish on $\mathcal{W}$.

If we are using the standard inner product on $\mathbb{R}^{n}$, then we can characterize adapted normals more concretely. 


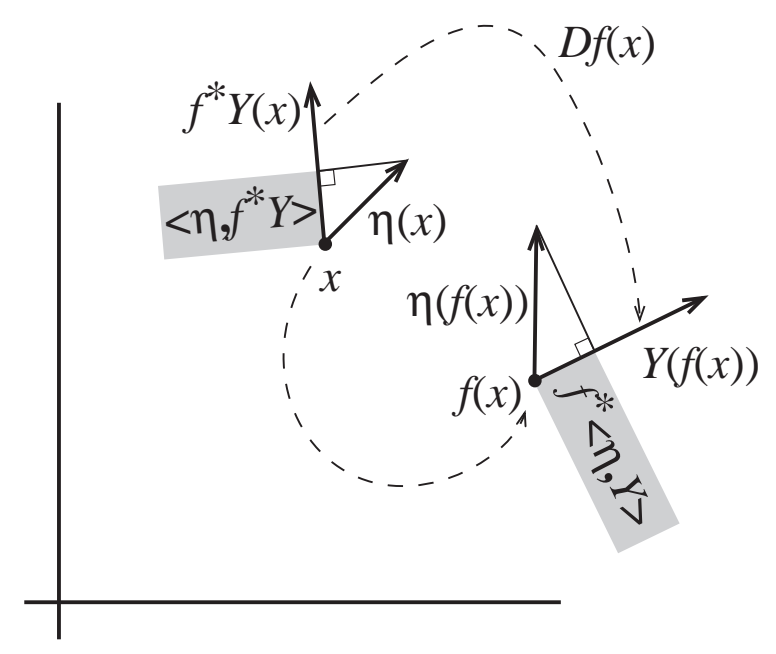

Figure 1: $\eta$ is an adapted normal if the two rectangles shown have the same area.

Proposition 2. Let $\eta: \mathcal{W} \rightarrow \mathbb{R}^{n}$ be a smooth function defined on the invariant surface $\mathcal{W}$, and suppose $\langle u, v\rangle=u^{t} \cdot v$ is the standard inner product on $\mathbb{R}^{n}$. Then $\eta$ satisfies (3) for all vector fields $Y: \mathcal{W} \rightarrow \mathbb{R}^{n}$ if and only if, for all $x \in \mathcal{W}$

$$
D f(x)^{t} \eta(f(x))=\eta(x)
$$

In the general case $\mathcal{W}$ is not defined as the level set of an invariant, and it is not easy to show that an adapted normal field exists. However, when the map is volume-preserving and we are given an appropriate parameterization of the invariant surface, an adapted normal vector field can easily be constructed.

Lemma 3. Suppose that $f: \mathbb{R}^{n} \rightarrow \mathbb{R}^{n}$ preserves the volume-form $\Omega$, and has a smooth invariant surface $\mathcal{W}$. Suppose $k: \mathbb{R}^{n-1} \rightarrow \mathbb{R}^{n}$ is a nondegenerate parameterization of $\mathcal{W}$ with the property

$$
f(k(u))=k(u+\delta),
$$

for a constant $\delta \in \mathbb{R}^{n-1}$. Then the vector field $\eta: \mathcal{W} \rightarrow \mathbb{R}^{n}$ restricted to $\mathcal{W}$ defined by

$$
\langle\eta, \cdot\rangle=\Omega\left(\cdot, \partial_{u_{1}} k, \partial_{u_{2}} k, \ldots, \partial_{u_{n-1}} k\right)
$$

is an adapted vector field on $\mathcal{W}$.

Proof. The nondegeneracy of the parameterization implies that $\eta \neq 0$ on the surface $\mathcal{W}$. Condition (5) implies that

$$
D_{u} k(u+\delta)=D_{u}(f \circ k)(u)=D_{x} f(k(u)) D_{u} k(u) ;
$$


therefore, since $f^{*} \Omega=\Omega$, we have

$$
\begin{aligned}
f^{*}\langle\eta(k), Y(k)\rangle & =\langle\eta(f(k)), Y(f(k))\rangle \\
& =\Omega_{f(k)}\left(Y(f(k)), \partial_{u_{1}} k(u+\delta), \ldots, \partial_{u_{n-1}} k(u+\delta)\right) \\
& =\Omega_{f(k)}\left(Y(f(k)), D f(k) \partial_{u_{1}} k(u), \ldots, D f(k) \partial_{u_{n-1}} k(u)\right) \\
& =\Omega_{x}\left(D f(x)^{-1} Y(f(k)), \partial_{u_{1}} k(u), \ldots, \partial_{u_{n-1}} k(u)\right) \\
& \left.=\left\langle\eta(k), f^{*} Y\right)\right\rangle . \quad \square
\end{aligned}
$$

\subsection{Adapted one-forms}

An alternative concept to that of adapted vectors are adapted forms. The advantage of one versus the other approach is mainly a question of taste, though differential forms can be used without assuming an inner product. We now define an "adapted one-form."

Definition (Adapted one-form). Suppose that $f: \mathbb{R}^{n} \rightarrow \mathbb{R}^{n}$ is a diffeomorphism. An adapted one-form on an invariant surface $\mathcal{W}$ is a smooth function $\nu: T_{\mathcal{W}} \mathbb{R}^{n} \rightarrow \mathbb{R}$ such that

- $\nu_{x}$ is nondegenerate for all $x \in \mathcal{W}$.

- $\nu_{x}(v)=0$ for all $v \in T_{x} \mathcal{W}$.

- $f^{*} \nu=\nu$

Note that when $\nu$ is an adapted one-form then for each $x \in \mathcal{W}, \operatorname{ker}\left(\nu_{x}\right)=T_{x} \mathcal{W}$, but that since $\nu$ is nondegenerate, it will not be zero for vectors that are not tangent to $\mathcal{W}$. As before, we note that if $\mathcal{W}$ is given as the level surface of an invariant function $J$, i.e. if $J(f(x))=J(x)$, then an adapted one-form is easy to obtain: the one-form $d J$ is adapted provided only that $J$ has no critical points on $\mathcal{W}$. This follows because $f^{*} d J=d(J \circ f)=d J$.

Given an inner product, $\langle\cdot, \cdot\rangle$ we can always associate a unique vector field, $\eta$ with a form $\nu$, through $i_{X} \nu=\nu(X)=\langle\eta, X\rangle$. Here $\eta: \mathcal{W} \rightarrow \mathbb{R}^{n}$ is a smooth function. It is easy to see that, if the $\eta$ is adapted, then $\nu$ is also adapted. Conversely, given an adapted one-form, we can find an adapted normal field, through the same relation.

Proposition 4. Let $\eta$ and $\nu$ be related through

$$
i_{X} \nu=\langle\eta, X\rangle .
$$

Then $\eta$ is an adapted normal if and only if $\nu$ is an adapted form.

Using this with Lem. 3 implies immediately.

Corollary 5. Let $f: \mathbb{R}^{n} \rightarrow \mathbb{R}^{n}$ preserve a volume-form $\Omega$, and $\mathcal{W}$ be a smooth invariant surface. Suppose $k: \mathbb{R}^{n-1} \rightarrow \mathbb{R}^{n}$ is a nondegenerate parameterization of $\mathcal{W}$ such that $f(k(u, v))=k(u+\delta)$ for constant $\delta \in \mathbb{R}^{n-1}$. Then

$$
\nu=\Omega\left(\cdot, \partial_{u_{1}} k, \ldots, \partial_{u_{n-1}} k\right)
$$

is an adapted one-form on $\mathcal{W}$. 


\subsection{Example}

Let $f: \mathbb{R}^{3} \rightarrow \mathbb{R}^{3}$ be the two parameter family of diffeomorphisms

$$
f(x, y, z)=\left(\begin{array}{c}
e^{\tau}(x \cos \theta-y \sin \theta) \\
e^{\tau}(x \sin \theta+y \cos \theta) \\
\frac{1}{2}\left(x^{2}+y^{2}\right)+e^{-2 \tau} z
\end{array}\right),
$$

where $\tau$ and $\theta$ are constants. It is easy to see that $f$ preserves the standard volume-form $\Omega=d x \wedge d y \wedge d z$. In addition, $f$ has an invariant surface given by

$$
\mathcal{W}=\left\{(x, y, z) \in \mathbb{R}^{n} \backslash\{0\}: x^{2}+y^{2}=4 z \sinh (2 \tau)\right\} ;
$$

however, the "obvious" function $x^{2}+y^{2}-4 z \sinh (2 \tau)$ is not invariant. Instead, we parameterize $\mathcal{W}$ with the function $k: \mathbb{R}^{2} \rightarrow \mathbb{R}^{3}$ given by

$$
k(u, v)=\left(\begin{array}{c}
e^{u \tau} \cos v \\
e^{u \tau} \sin v \\
(4 \sinh 2 \tau)^{-1} e^{2 u \tau}
\end{array}\right) .
$$

The function $k$ is nondegenerate and satisfies

$$
f(k(u, v))=k(u+1, v+\theta) .
$$

so that Lem. 3 and Lem. 5 apply. Using (8), we find that $\nu=-2 \tau z(x d x+y d y-$ $2 \sinh (2 \tau) d z)$ an adapted form on $\mathcal{W}$. In other words $f^{*} \nu=\nu$ and $\operatorname{ker} \nu_{p}=T_{p} \mathcal{W}$, as can be explicitly verified. Also using (6), it is possible to show that

$$
\eta(x, y, z)=\left(\begin{array}{c}
-2 \tau x z \\
-2 \tau y z \\
4 \tau z \sinh 2 \tau
\end{array}\right)
$$

is an adapted normal field on $\mathcal{W}$. In other words, it satisfies $D f(x)^{t} \eta(f(x))=\eta(x)$, for each $x \in \mathcal{W}$. Note that $\nu$ and $\eta$ are related through $i_{X} \nu=\langle\eta, X\rangle$.

\section{Melnikov Function}

Suppose that the diffeomorphism $f_{0}$ has two normally hyperbolic invariant sets $p$ and $q$, and a codimension-one surface $\mathcal{W}=W^{u}(p)=W^{s}(q)$ that is a saddle connection between them. Upon perturbation, suppose that the corresponding invariant sets $p_{\varepsilon}$ and $q_{\varepsilon}$ of $f_{\varepsilon}$ have a stable manifold $\mathcal{W}_{\varepsilon}^{s}$ and unstable manifold $\mathcal{W}_{\varepsilon}^{u}$. Then in our notation, the classical Melnikov function is the smooth function $M_{\nu}: \mathcal{W} \rightarrow \mathbb{R}$ on the saddle connection $\mathcal{W}$ defined by

$$
M_{\nu} \equiv \nu\left(\left.\frac{\partial}{\partial \varepsilon}\right|_{\varepsilon=0}\left(\phi_{\varepsilon}^{u}-\phi_{\varepsilon}^{s}\right)\right),
$$


for a given adapted form $\nu$ on $\mathcal{W}$, and a given pair of adapted perturbations $\phi_{\varepsilon}^{s}$ and $\phi_{\varepsilon}^{u}$ corresponding to the stable and unstable manifolds, respectively. Thus $M_{\nu}$ measures the relative "velocity" of the manifolds as a function of $\varepsilon$. While $M_{\nu}$ appears to depend on the choice of adapted perturbations, we will show that it does not.

A function similar to $M_{\nu}$ was used in [22], to study the topology of heteroclinic connections of fixed points. Our purpose is to apply the method to the case of invariant circles, as illustrated in Fig. 2.

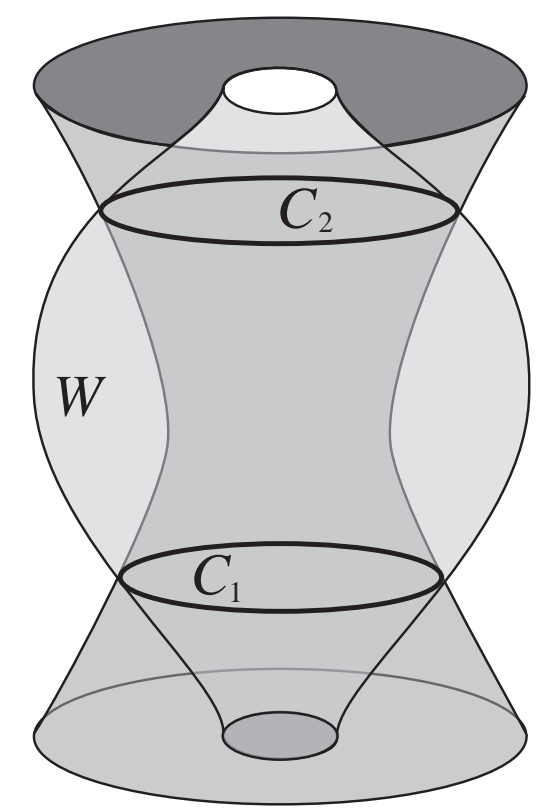

Figure 2: Two normally hyperbolic invariant circles $C_{1}$ and $C_{2}$ with a saddle connection $\mathcal{W}$.

\subsection{The fundamental iterative relation}

The fundamental relation used in deriving the Melnikov function is an iteration formula obtained by combining the definition of adapted one-form and Prop. 1 .

Theorem 6. Suppose $f_{\varepsilon}$ is a family of diffeomorphisms with invariant surfaces $\mathcal{W}_{\varepsilon}$. Let $\nu$ be an adapted one-form and $\phi_{\varepsilon}$ be an adapted deformation on $\mathcal{W}_{0}$. Define $\mu: \mathcal{W} \rightarrow \mathbb{R}$ by

$$
\mu(x)=\nu\left(\left.\partial_{\varepsilon} \phi(x, \varepsilon)\right|_{\varepsilon=0}\right) .
$$

Then

$$
\mu-\mu \circ f_{0}^{-1}=\nu\left(X_{0}\right)
$$

where $X_{0}$ is the perturbation vectorfield, (1). Moreover, if $\tilde{\phi}_{\varepsilon}$ is another adapted deformation and $\tilde{\mu}$ is defined similarly to $\mu$, then $\mu=\tilde{\mu}$. 
Proof. By Prop. 目 the difference between the derivatives of two adapted diffeomorphisms is tangent to $\mathcal{W}$, and by Defn. 2.3, $\nu$ vanishes on any tangent vector. Thus $\mu=\tilde{\mu}$. To compute the second relation, use $f_{0}^{*} \nu=\nu$ to find

$$
\begin{aligned}
\mu & =f_{0}^{*} \nu_{x}\left(\partial_{\varepsilon} \phi_{\varepsilon}(x)\right) \\
& =\nu_{f_{0}(x)}\left(D f_{0}(x) \partial_{\varepsilon} \phi_{\varepsilon}(x)\right) \\
& =\nu_{f_{0}(x)}\left(\partial_{\varepsilon} f_{\varepsilon}\left(\phi_{\varepsilon}(x)\right)-\partial_{\varepsilon} f_{\varepsilon}(x)\right),
\end{aligned}
$$

where we suppress the $\varepsilon=0$ expressions for simplicity. Using (11) $X_{0}=\partial_{\varepsilon} f_{\varepsilon}\left(f_{0}^{-1}(x)\right)$, we have $\mu \circ f_{0}^{-1}=\nu_{x}\left(\partial_{\varepsilon} f_{\varepsilon}\left(\phi_{\varepsilon}\left(f_{0}^{-1}(x)\right)\right)\right)-\nu_{x}\left(X_{0}\right)$, and therefore

$$
\mu-\mu \circ f_{0}^{-1}=\nu\left(\partial_{\varepsilon} \phi-\partial_{\varepsilon} f_{\varepsilon}\left(\phi_{\varepsilon}\left(f_{0}^{-1}(x)\right)\right)\right)+\nu\left(X_{0}\right) .
$$

Noting that $\tilde{\phi}_{\varepsilon}=f_{\varepsilon}\left(\phi_{\varepsilon}\left(f_{0}^{-1}(x)\right)\right.$ is also an adapted diffeomorphism, we see that the first term vanishes by Prop. 囵.

Equation (10) gives us a recursive formula to compute the normal component of the change in the manifold $\mathcal{W}_{\varepsilon}$.

Corollary 7. Under the assumptions of Thm. G, for all $n \in \mathbb{N}$

$$
\mu=\mu \circ f_{0}^{-n}+\sum_{k=0}^{n-1} \nu\left(X_{0}\right) \circ f_{0}^{-k} .
$$

In addition, if $\lim _{n \rightarrow \infty} \mu \circ f_{0}^{-n}(x)=0$, then

$$
\mu(x)=\sum_{k=0}^{\infty} \nu\left(X_{0}\right) \circ f_{0}^{-k}=\sum_{k=0}^{\infty} \nu\left(\left(f_{0}^{-k}\right)^{*} X_{0}\right) .
$$

These statements can be directly transcribed for adapted normals using Prop. 因

\subsection{Transversal intersections}

According to Thm. 6 and Cor. 0, we can compute the Melnikov function (9) in terms of the first order perturbation vector field $X_{0}$.

Proposition 8. Suppose $f$ has a codimension-one saddle connection $\mathcal{W}$ between two normally hyperbolic invariant sets $p$ and $q$. Assume that for all $x \in p \cup q$, the perturbation vector field $X_{0}(x)=0$. Let $\nu$ be an adapted form and $\eta$ the corresponding adapted normal defined on $\mathcal{W}$. Define the Melnikov function by

$$
M_{\nu}=\sum_{k=-\infty}^{\infty} \nu\left(X_{0}\right) \circ f_{0}^{k}=\sum_{k=-\infty}^{\infty}\left\langle\eta, X_{0}\right\rangle \circ f_{0}^{k} .
$$

Then if a point $x_{0} \in \mathcal{W}$ is a nondegenerate zero of $M_{\nu}$, the stable and unstable manifolds $W^{u}\left(q, f_{\varepsilon}\right)$ and $W^{s}\left(p, f_{\varepsilon}\right)$ intersect transversally near $x_{0}$ for $\varepsilon$ small enough. 
Proof. For each point $x$ in the saddle connection $\mathcal{W}$, there is a neighborhood $\mathcal{N}_{0} \subset \mathcal{W}$, such that all the iterates $f^{k}\left(\mathcal{N}_{0}\right)$ are disjoint. Moreover, since $p$ and $q$ are normally hyperbolic, the stable manifold theorem implies that there is an $\varepsilon_{0}>0$ such that there exist adapted deformations $\phi^{u}: \mathcal{N}_{0} \times\left(-\varepsilon_{0}, \varepsilon_{0}\right) \rightarrow W^{u}\left(q, f_{\varepsilon}\right)$, and $\phi^{s}: \mathcal{N}_{0} \times\left(-\varepsilon_{0}, \varepsilon_{0}\right) \rightarrow W^{s}\left(p, f_{\varepsilon}\right)$.

Consider first the unstable part. Let $\mathcal{V}=\bigcup_{k=0}^{\infty} f_{0}^{-k}\left(\mathcal{N}_{0}\right)$. Clearly $\mathcal{V}$ is a immersed manifold. Moreover, we can extend the domain of $\phi^{u}$ to all of $\mathcal{V}$, by defining

$$
\phi^{u}(x, \varepsilon)=f_{\varepsilon}^{-k}\left(\phi^{u}\left(f_{0}^{k}(x), \varepsilon\right)\right),
$$

provided that $x \in f_{0}^{-k}\left(\mathcal{N}_{0}\right)$. It is clear that for each $\varepsilon \in\left(-\varepsilon_{0}, \varepsilon_{0}\right)$ and $x \in \mathcal{V}$, we have that $\phi^{u}(x, \varepsilon) \in W^{u}\left(q, f_{\varepsilon}\right)$.

For each $x$, we are interested in estimating $\phi(x, \varepsilon)$ to first order in $\varepsilon$. Using $\phi^{u}$ in Cor. 7 gives (11) providing $\mu^{u} \circ f^{-n}(x) \rightarrow 0$. This is the case because $\phi^{u}\left(f^{-n}(x), \varepsilon\right) \rightarrow 0$ so that $\partial_{\varepsilon} \phi^{u}$ is bounded, and $\nu_{f_{0}^{-n}(x)} \rightarrow 0$ since it is an adapted form.

Similar analysis applies to the stable adapted deformation, and again Cor. 7 applies, though we iterate in the opposite direction, to obtain $\mu^{s}=-\sum_{k=1}^{\infty} \nu\left(X_{0}\right) \circ f_{0}^{k}$. According to (9), the difference between $\mu^{u}$ and $\mu^{s}$ gives the Melnikov function, which yields (12).

Following a standard Melnikov argument based on the implicit function theorem [25], we conclude that if $x_{0}$ is a nondegenerate zero $M_{\nu}$ then near $x_{0}$, the two manifolds $W^{u}\left(f_{\varepsilon}\right)$ and $W^{s}\left(f_{\varepsilon}\right)$ intersect transversely.

\section{Flux}

The flux across a surface is the volume that crosses the surface each iterate of a map; it is an important measure of transport. Recall that for area-preserving maps, the Melnikov function is a measure of the distance between the stable and unstable manifolds, and that its integral between two successive zeros is the geometric flux that crosses the "separatrix" each iteration of the map [15, 16]. The outgoing flux is exactly balanced by an ingoing flux, so that the net, or algebraic, flux crossing the separatrix is zero.

Here we will obtain an analogous formula for volume-preserving maps (see also [8]). We start by constructing a flux form on an invariant set. We will see that the algebraic flux crossing the separatrix is zero. This implies, for example that the Melnikov function has zeros in the separatrix.

\subsection{Flux Form}

It is well known that a volume-preserving map with an invariant $J$ can be restricted to a measure preserving map on any surface $J=c$ on which $\nabla J$ is nonzero. That is, the form $\omega=|\nabla J|^{-2} i_{\nabla J} \Omega$ is an invariant $n-1$ form for the map $\left.f\right|_{J=c}$. We show here that a similar preserved measure also exists if we can find an adapted normal for an invariant surface $\mathcal{W}$. We will then use this to construct a flux form on $\mathcal{W}$.

As usual, we assume that $f$ is a diffeomorphism with an invariant volume-form $\Omega, \mathcal{W}$ is an invariant codimension-one hypersurface, and $\langle$,$\rangle is an inner product on \mathbb{R}^{n}$. 
Proposition 9. Suppose $\eta$ is an adapted normal field on $\mathcal{W}$. Then

$$
\omega_{\eta}=\frac{i_{\eta} \Omega}{\langle\eta, \eta\rangle}
$$

is a nondegenerate $(n-1)$-form on $\mathcal{W}$ that is invariant under the restricted map $\left.f\right|_{\mathcal{W}}$ : $\mathcal{W} \rightarrow \mathcal{W}$.

Proof. It is clear that $\omega_{\eta}$ is a nondegenerate $(n-1)$-form on $\mathcal{W}$. We need to show that $f_{0}^{*} \omega_{\eta}=\omega_{\eta}$ for vectors in $T \mathcal{W}$. With some manipulations we have

$$
\begin{aligned}
f^{*} \omega_{\eta}-\omega_{\eta} & =\frac{i_{f^{*} \eta} f^{*} \Omega}{f^{*}\langle\eta, \eta\rangle}-\frac{i_{\eta} \Omega}{\langle\eta, \eta\rangle} \\
& =\frac{i_{f^{*} \eta} \Omega}{\left\langle\eta, f^{*} \eta\right\rangle}-\frac{i_{\eta} \Omega}{\langle\eta, \eta\rangle}=i_{v} \Omega
\end{aligned}
$$

where we define the vector field:

$$
v=\frac{f^{*} \eta}{\left\langle\eta, f^{*} \eta\right\rangle}-\frac{\eta}{\langle\eta, \eta\rangle}
$$

Since $\langle\eta, v\rangle=0$ on $\mathcal{W}$ for each point $x \in \mathcal{W}$, and $\eta$ defines the normal direction, then $v(x) \in T_{x} \mathcal{W}$. Since $v$ is tangent to $\mathcal{W}$, the form $i_{v} \Omega$ restricted to $\mathcal{W}$ has to vanish. Thus, we conclude that $\left(f^{*} \omega_{\eta}-\omega_{\eta}\right)_{\mathcal{W}}=0$.

From now on assume that $\mathcal{W}$ has an adapted vector field $\eta$, and the Melnikov sum (12) exists. We then define

Definition (Flux Form). The flux form $\Phi \equiv M_{\eta} \omega_{\eta}$ is an $(n-1)$-form on $\mathcal{W}$.

Note that $\Phi$ might be degenerate (it has zeros), and since the space of $(n-1)$-forms on the $(n-1)$-dimensional manifold $\mathcal{W}$ is one-dimensional, it might not be unique. However, this is not the case:

Lemma 10. If the Melnikov function exists, then the form $\Phi$ is independent of the choice of $\eta$.

Proof. The projection of $X_{0}$ onto $T \mathcal{W}$ is the vector $v=X_{0}-\frac{\left\langle\eta, X_{0}\right\rangle}{\langle\eta, \eta\rangle} \eta$. Note that

$$
i_{X_{0}} \Omega-\left\langle\eta, X_{0}\right\rangle \omega_{\eta}=i_{v} \Omega
$$

Since $v \in T \mathcal{W}$, we conclude that $i_{v} \Omega=0$, as a $(n-1)$-form in the surface $\mathcal{W}$, and so we have

$$
\left\langle\eta, X_{0}\right\rangle \omega_{\eta}=i_{X_{0}} \Omega
$$

for all vectors in $T \mathcal{W}$. This implies that the summand of $M_{\eta} \omega_{\eta}$, recall (12), can be rewritten

$$
\left(f_{0}^{k}\right)^{*}\left\langle\eta, X_{0}\right\rangle \omega_{\eta}=\left(f_{0}^{k}\right)^{*} i_{X_{0}} \Omega
$$

which is independent of $\eta$. 
Thus we have

$$
\Phi=M_{\eta} \omega_{\eta}=\sum_{k=-\infty}^{\infty}\left(f_{0}^{k}\right)^{*} i_{X_{0}} \Omega .
$$

Since $\omega_{\eta}$ is nondegenerate, the degenerate points of the flux form correspond to zeros of the Melnikov function. As we will see in $\S 4.3$, the integral of the flux form over a piece of $\mathcal{W}$ gives flux through that surface to first order in $\varepsilon$.

The form $i_{X_{\varepsilon}} \Omega$ has some interesting properties:

Proposition 11. Let $X_{\varepsilon}$ be a perturbation vector field (1). Then the form $i_{X_{\varepsilon}} \Omega$ is exact.

Proof. As we already noted,Thm 2.2.24 in [24] implies that the divergence of $X_{\varepsilon}$ vanishes, which by definition $\left(\operatorname{div}_{\Omega} X_{\varepsilon}\right) \Omega \equiv L_{X_{\varepsilon}} \Omega$, means that the Lie derivative vanishes as well. Since $d \Omega=0$ and $L_{X_{\varepsilon}} \Omega \equiv d\left(i_{X_{\varepsilon}} \Omega\right)+i_{X_{\varepsilon}} d \Omega$, this implies that $d\left(i_{X_{\varepsilon}} \Omega\right)=0$. Thus the form is closed. Since $i_{X_{\varepsilon}} \Omega$ is globally defined in $\mathbb{R}^{n}$, the form is exact.

Using this result we can obtain an $(n-2)$-form $\beta$ on $\mathcal{W}$ such that

$$
d \beta \equiv i_{X_{0}} \Omega
$$

In this case we will say that $\beta$ represents the perturbation on $\mathcal{W}$. Using Lem. 10 and (14), it is easy to see that if $M_{\eta}$ exists then the following $(n-2)$-form is well-defined on $\mathcal{W}$.

$$
\alpha=\sum_{k=-\infty}^{\infty}\left(f_{0}^{k}\right)^{*} \beta .
$$

Notice that $\alpha$ is invariant under $f$ and is independent of $\eta$. Using this we can see that:

Proposition 12. The flux form $\Phi$ is

$$
\Phi=d \alpha
$$

Proof. This is a straightforward calculation using Lem. 10 and Prop. 9.

\subsection{Fundamental Domains}

Our goal in this section is to find a compact subset of the manifold - a fundamental domain - that generates the entire manifold under iteration by $f_{0}$. We will integrate the flux form over the fundamental domain to show that the algebraic flux crossing the separatrix is zero. From this point on, we will concentrate on the case $n=3$. To define the fundamental domain we start with the concept of a proper loop:

Definition (Proper loop). Let $f_{0}: \mathbb{R}^{3} \rightarrow \mathbb{R}^{3}$ be a diffeomorphism, and $\mathcal{W}$ a forward invariant surface. We say that a smooth Jordan loop $\gamma \subset \mathcal{W}$ is a proper boundary in $\mathcal{W}$ if there $\gamma$ bounds a surface $\mathcal{W}_{\gamma} \subset \operatorname{int}(\mathcal{W})$ which is a trapping region:

$$
f\left(\operatorname{cl}\left(\mathcal{W}_{\gamma}\right)\right) \subset \operatorname{int}\left(\mathcal{W}_{\gamma}\right)
$$


Similarly a loop $\gamma$ is a proper loop for a backward invariant surface if it is a proper loop for the map $f^{-1}$.

It is important to notice that not all invariant surfaces admit proper boundaries. A trivial observation is:

Proposition 13. If $\gamma$ is a proper boundary in $\mathcal{W}$, then $f(\gamma)$ is also a proper boundary. In addition, $\mathcal{W}_{f(\gamma)}=f\left(\mathcal{W}_{\gamma}\right)$.

The situation that we have in mind relates to the structure of stable and unstable manifolds. Let $a, b$ be compact, normally hyperbolic invariant sets of $f$, and $\mathcal{W}=W^{s}(a)=$ $W^{u}(b)$ a saddle connection between them. A proper loop $\gamma \subset \mathcal{W}$ is a submanifold of $\mathcal{W}$ that bounds a local submanifold that is a isolating neighborhood of $a$ in $W^{s}(a)$. In other words $\gamma$ is proper if it bounds an open local submanifold, $W_{l o c}^{s}(a)=\mathcal{W}_{\gamma}$, that maps inside itself.

If $\gamma$ is proper, we can define the stable manifold starting at $\gamma$, denoted by $\mathcal{W}_{\gamma}=W_{\gamma}^{s}(a)$, as the closure in $W^{s}(a)$ of the local stable manifold bounded by $\gamma$. In the same way, for $b$, if we have a proper loop $\sigma$ for $f^{-1}$, we define the unstable manifold up to $\sigma$, denoted $W_{\sigma}^{u}(b)$, as the interior of the local unstable manifold bounded by $\sigma$. We will see below why it is convenient to use this slightly asymmetric definition.

Given a proper loop we can define

Definition (Fundamental domain). Let $\mathcal{W}$ be a forward invariant surface. An submanifold with boundary, $\mathcal{P}$, is a fundamental domain of $\mathcal{W}$ if there exists some proper loop $\gamma$ in $\mathcal{W}$, such that

$$
\mathcal{P}=\mathcal{P}_{\gamma}=\mathcal{W}_{\gamma} \backslash \mathcal{W}_{f(\gamma)}
$$

The fundamental domain is a manifold with the boundary

$$
\partial \mathcal{P}=\gamma \cup f(\gamma)
$$

see Fig. 3. An immediate consequence of the definition is that all the forward iterations of a fundamental domain are also fundamental and $\mathcal{P}_{f(\gamma)}=f\left(\mathcal{P}_{\gamma}\right)$. It is easy to see that, if proper boundaries exist, then the forward invariant manifold can be decomposed as the disjoint union of fundamental domains.

$$
\mathcal{W}=\left(\mathcal{W} \backslash \mathcal{W}_{\gamma}\right) \cup \bigcup_{k \geq 0} f^{k}(\mathcal{P})
$$

If the surface $\mathcal{W}$ is both forward and backward invariant, then this decomposition works in both directions. In such case we have

$$
\mathcal{W}=\bigcup_{k \in \mathbb{Z}} f^{k}(\mathcal{P})
$$




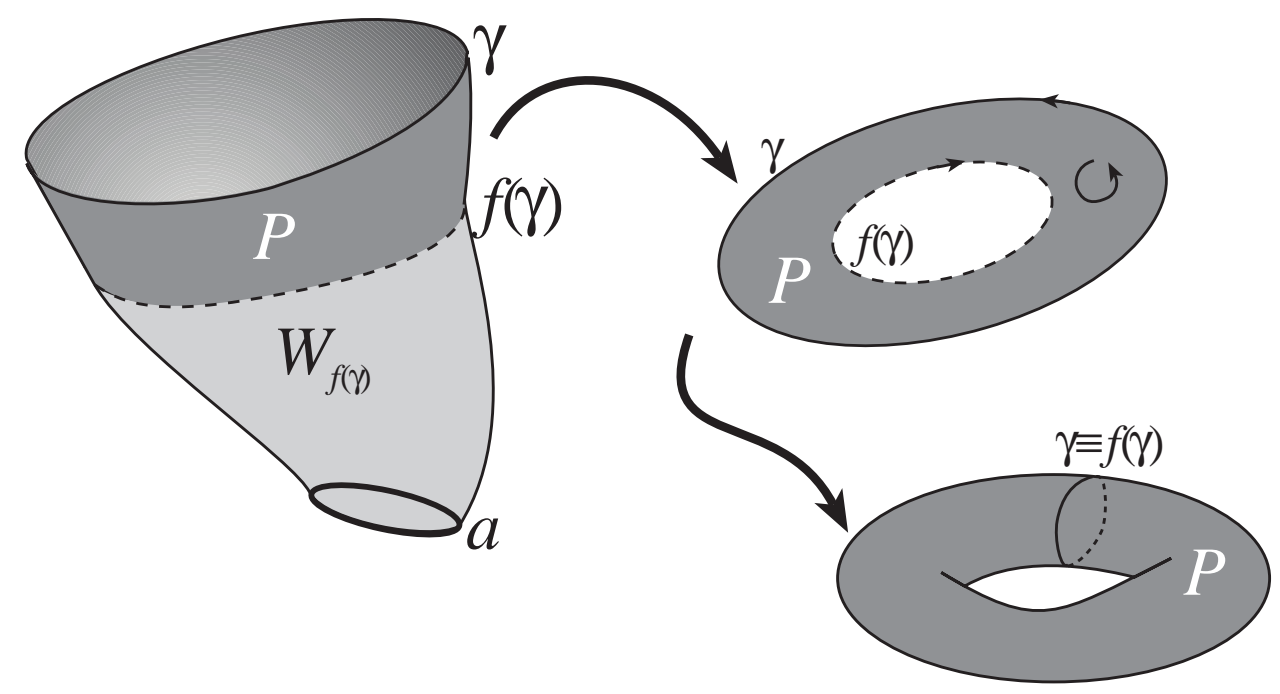

Figure 3: Fundamental domain $\mathcal{P}$ on a stable manifold of an invariant circle $a$ bounded by a loop $\gamma$ and its image $f(\gamma)$. The second part of the figure shows the annular fundamental domain itself, together with an assigned orientation. Finally, if we identify the points on $\gamma$ with their images, then the fundamental domain is equivalent to a torus.

\subsection{Algebraic Flux}

Given a vector field $X$, the differential form $i_{X} \Omega$ represents the flux associated with $X$; that is given set of vectors $v_{1}, v_{2}, \ldots v_{n-1}, \Omega\left(X, v_{1}, v_{2}, \ldots v_{n-1}\right)$ is the volume of the parallelepiped formed from these vectors, and thus measures the rate at which volume is swept out by $X$ through the parallelepiped defined by $v_{1}, v_{2}, \ldots v_{n-1}$.

According to (13), the form $\Phi$ is the sum of $i_{X_{0}} \Omega$ along an orbit on $\mathcal{W}$. Thus $\Phi$ evaluated at a point on a fundamental domain $\mathcal{P}$ measures the total flux of $X_{0}$ along the orbit of that point.

The algebraic flux through a surface is the integral of the flux over the surface. Since $\Phi$ measures the flux along an orbit on $\mathcal{W}$, the integral of $\Phi$ over a fundamental domain is the algebraic flux through the entire surface $\mathcal{W}$.

Proposition 14. The algebraic flux through $\mathcal{W}$ is zero: $\int_{\mathcal{W}} i_{X_{0}} \Omega=\int_{\mathcal{P}} \Phi=0$.

Proof. The fundamental domain $\mathcal{P}=\mathcal{P}_{\gamma}$ is a submanifold with boundary, such that $\partial \mathcal{P}_{\gamma}=\gamma \cup f(\gamma)$, where $\gamma$ is closed curve that does not intersect $f(\gamma)$. If we give an orientation $[\mathcal{P}]$ to $\mathcal{P}$, the induced orientation on the boundary satisfies $[\gamma]=-[f(\gamma)]$, recall Fig. 3. Since $\Phi=d \alpha$ by Prop. 12, and $\alpha$ is invariant under $f$, Stokes's theorem, implies

$$
\begin{aligned}
\int_{\mathcal{P}} \Phi & =\int_{\mathcal{P}} d \alpha=\int_{\partial \mathcal{P}} \alpha=\int_{\gamma} \alpha+\int_{f(\gamma)} \alpha \\
& =\int_{\gamma} \alpha-\int_{\gamma} f^{*} \alpha=0
\end{aligned}
$$


A simple corollary is

Corollary 15. The Melnikov function must have zeros on $\mathcal{P}$.

The importance of fundamental domains is that much of the information about the entire manifold can be found by looking only at these submanifolds. In particular, if we have a transversal intersection of two invariant surfaces, we can look at a pair of fundamental domains and study primary intersections. In Fig. 团we show a pair of fundamental domains of two stable and unstable manifolds that intersect transversally.

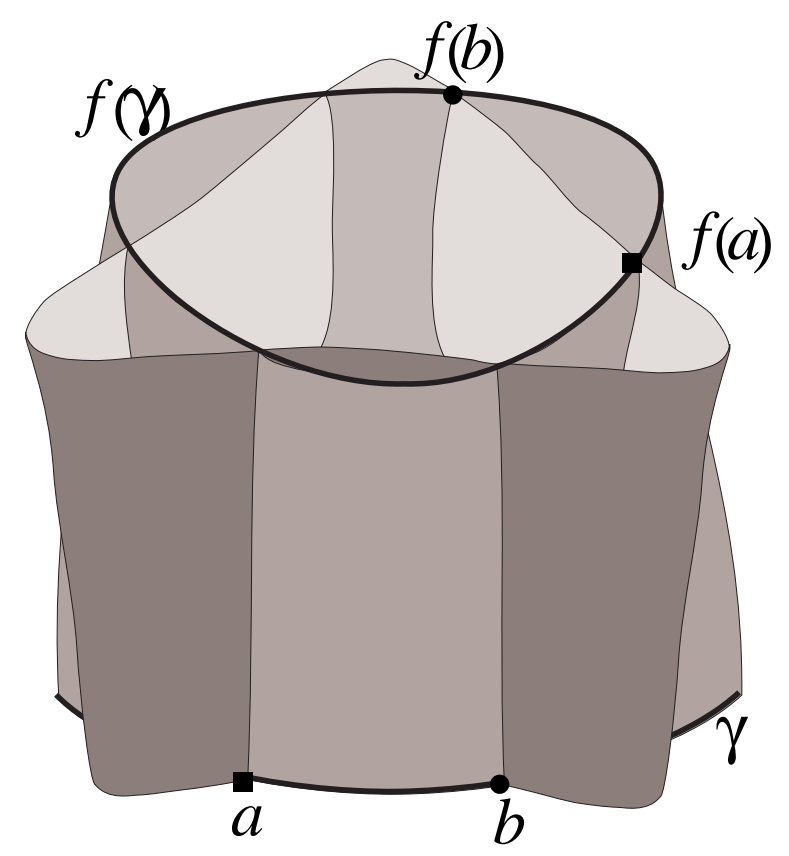

Figure 4: A pair of fundamental domains for two different circles $C_{1}$ and $C_{2}$ that intersect transversally, forming three-dimensional lobes.

The curves of zeros of the Melnikov function can be classified by their homology on $\mathcal{P}$. To do this, we identify two boundaries of the fundamental annulus by identifying $\gamma$ with $f(\gamma)$. With this identification the fundamental annulus becomes a torus, as sketched in Fig. 3. Since the homology group of the torus is $\mathbb{Z}^{2}$, we can label the curves by a pair of integers $(m, n)$ which represent the number of times the curves wrap around each circuit of the torus. For example when the identification is performed on Fig. 1 there are a pair of zero crossing curves with homology type $(3,1)$ - they move once around the annulus in three vertical transits.

\section{$5 \quad$ Examples}

In this section we construct a family of volume-preserving maps that have a saddle connection between a pair of invariant circles. We obtain this family by starting with an 
area-preserving twist map that preserves an axis and extending it to a three-dimensional, volume-preserving map by composing it with a sheared rotation about that axis. The twist map is defined in such a way that it has a saddle connection between two fixed points, and so the resulting three-dimensional map has a pair of invariant circles with a two-dimensional connection. Examples similar to these were found by Lomelí [23] and are closely related to those in [22].

We begin with an area-preserving map on $\mathbb{R}^{2}$ in coordinates $(z, r)$ that preserves the axis $r=0$, and has a fixed point at some nonzero $r=r^{*}$. For example, set

$$
\left(r^{\prime}, z^{\prime}\right)=G(r, z)=\left(h^{-1}(r+h(z))-z, h(z)+r-r^{*}\right),
$$

where $r^{*} \in \mathbb{Z}$. Here we assume that $h: \mathbb{R} \rightarrow \mathbb{R}$ is an increasing circle diffeomorphism of period 1, i.e., $h(z+1)=h(z)+1$. Moreover we can verify that $\operatorname{det}(D G)=1$, so that $G$ is area-preserving. Finally

$$
G^{-1}(r, z)=\left(z-h\left(h^{-1}(z)-r\right), h^{-1}(z)+r^{*}-r\right),
$$

so that $G$ is a diffeomorphism.

It is easy to see that $G(0, z)=\left(0, h(z)-r^{*}\right)$ so that the $z$-axis is preserved. The map has fixed points at solutions of $z=\frac{1}{2}\left(h^{-1}(z)+h(z)\right)$, with $r=r^{*}+z-h(z)$. In particular, any hyperbolic fixed point of $h, z^{*}=h\left(z^{*}\right)$, yields a saddle fixed point $\left(r^{*}, z^{*}\right)$ of $G$ whose multipliers are $\lambda=h^{\prime}\left(z^{*}\right)$ and $1 / \lambda$. Between every pair of such fixed points of $h$ there is at least one other fixed point of $G$; it is typically elliptic.

The map $G$ is not necessarily integrable (in $\$ 5.1$ we will choose an $h$ that leads to an integrable map). However, $G$ always has a pair of invariant curves:

$$
\begin{aligned}
& \mathcal{W}_{0}=\left\{(z, r): r=r^{*}\right\}, \\
& \mathcal{W}_{1}=\left\{(z, r): r=h^{-1}(z)-h(z)+r^{*}\right\} .
\end{aligned}
$$

These curves intersect at any fixed point $z^{*}$ of $h$. Thus they provide a saddle connection between points $\left(z_{1}^{*}, r^{*}\right)$ and $\left(z_{2}^{*}, r^{*}\right)$, where $z_{i}^{*}$ are neighboring fixed points of $h$.

We show an example of the dynamics of $G$ in Fig. 同 for the case that $h(z)=z-$ $\frac{k}{2 \pi} \cos (2 \pi z)$, and $r^{*}=1.0$. Here one can see the saddle connection at $r=r^{*}$ as well as chaotic dynamics in other regions of phase space.

We can extend $G$ to $\mathbb{R}^{3}$ by introducing the cylindrical angle $\theta$ and using the volumeform $\Omega=d r \wedge d \theta \wedge d z$. Defining $r$ to be the "symplectic" polar radius,

$$
r=\frac{1}{2}\left(x^{2}+y^{2}\right),
$$

the cylindrical coordinates are

$$
(x, y, z)=P(r, \theta, z)=(\sqrt{2 r} \cos \theta, \sqrt{2 r} \sin \theta, z)
$$

so that $\Omega=d x \wedge d y \wedge d z$. In terms of these coordinates the map becomes $g=P \circ G \circ P^{-1}$ :

$$
g(x, y, z)=\left(\rho(r, z) x, \rho(r, z) y, r+h(z)-r^{*}\right),
$$




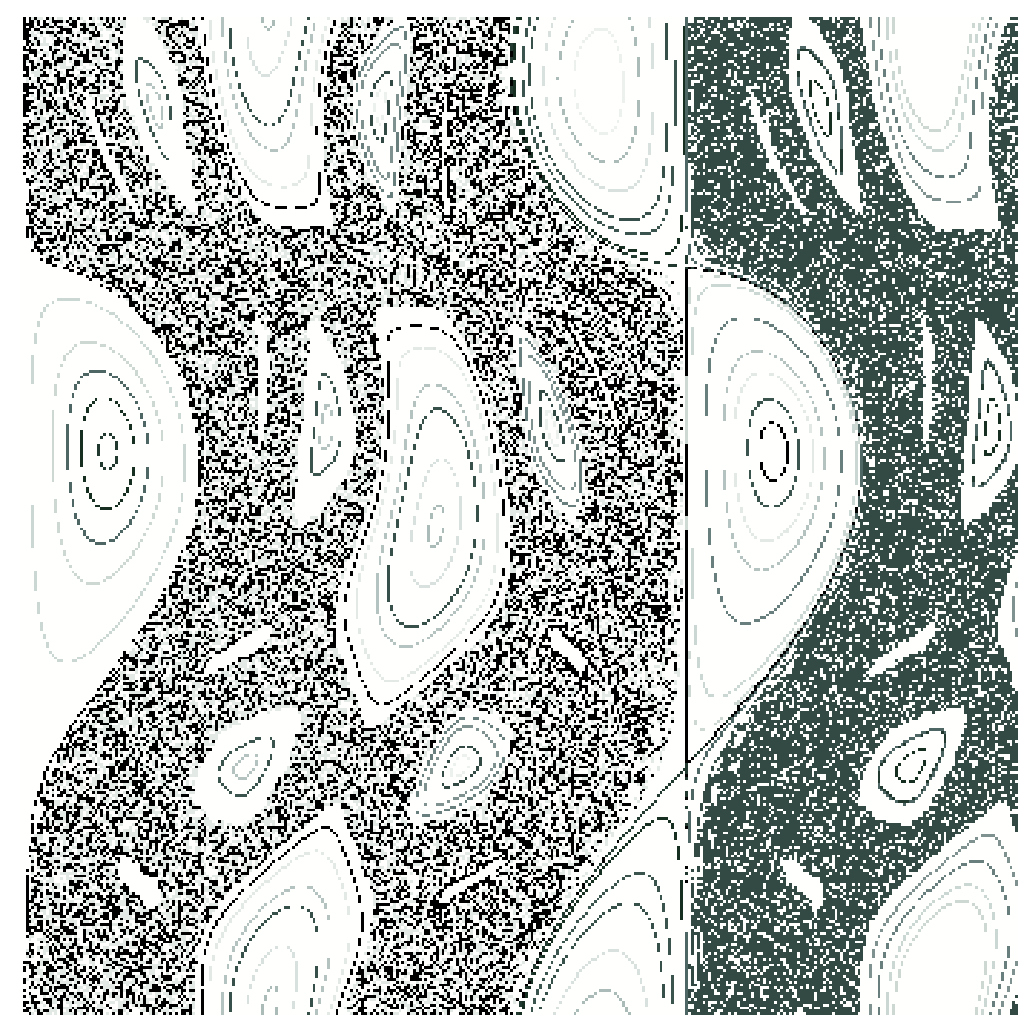

Figure 5: Dynamics of $G$ for the Arnold circle map with $k=0.9$. The domain of the figure is $[0,1.5] \times[-0.5,0.5]$.

where $\rho=\sqrt{r^{\prime} / r}$ is explicitly

$$
\rho(r, z)=\left\{\begin{array}{cc}
\sqrt{\frac{h^{-1}(r+h(z))-z}{r},} & r \neq 0 \\
\left(h^{\prime}(z)\right)^{-\frac{1}{2}}, & r=0 .
\end{array}\right.
$$

It was shown in [22] that if $h$ is $C^{r}$, then $\rho$ is $C^{r-1}$ so that, in this case, $g$ is a diffeomorphism.

The map becomes fully three-dimensional if we introduce dynamics in the angle $\theta$. To do this, we compose the map with a rotation. Denote the rotation about the $z$-axis by angle $\psi$ by

$$
R_{\psi}=\left(\begin{array}{ccc}
\cos \psi & -\sin \psi & 0 \\
\sin \psi & \cos \psi & 0 \\
0 & 0 & 1
\end{array}\right) .
$$

Introducing a rotation angle $\tau(r, z)$ that depends smoothly on $(r, z)$, we define a diffeomorphism $f$ by

$$
f=g \circ R_{\tau},
$$

Note that since $R_{\tau}$ and $g$ both preserve $\Omega$, so does $f$. 
The map $f$ still has a rotational symmetry

$$
f \circ R_{\psi}=R_{\psi} \circ f
$$

for any constant $\psi \in \mathbb{R}$. This implies that when $G$ has a saddle connection, so does $f$ :

Proposition 16. The surfaces (15) are invariant under (19). In addition, $\mathcal{W}_{0}$ and $\mathcal{W}_{1}$ intersect on the invariant circles

$$
\mathcal{C}\left(z^{*}\right)=\left\{(x, y, z): z=z^{*}, r=r^{*}\right\},
$$

where $z^{*}$ is any fixed point of $h$.

Every point on the circles $\mathcal{C}\left(z^{*}\right)$ is fixed under $g$. The derivative of $g$ at such points is

$$
D g(x, y, z)=\left(\begin{array}{ccc}
\frac{1}{2 r^{*}}\left(\lambda^{-1} x^{2}+y^{2}\right) & \frac{1}{2 r^{*}}\left(\lambda^{-1}-1\right) x y & 0 \\
\frac{1}{2 r^{*}}\left(\lambda^{-1}-1\right) x y & \frac{1}{2 r^{*}}\left(x^{2}+\lambda^{-1} y^{2}\right) & 0 \\
x & y & \lambda
\end{array}\right)
$$

where $\lambda=h^{\prime}\left(z^{*}\right)$. More generally, we can compute the derivative of $f^{n}$ on the invariant circles for the special case that the rotation angle is constant:

Proposition 17. Suppose $f$ is given by (19), that $\tau$ is constant, $z^{*}=h\left(z^{*}\right)$, and $\mathcal{C}\left(z^{*}\right)$ is the corresponding invariant circle. Then for all $(x, y, z) \in \mathcal{C}\left(z^{*}\right)$

$$
D f^{n}(x, y, z)=R_{n \tau}\left(\begin{array}{ccc}
\frac{1}{2 r^{*}}\left(\lambda^{-n} x^{2}+y^{2}\right) & \frac{1}{2 r^{*}}\left(\lambda^{-n}-1\right) x y & 0 \\
\frac{1}{2 r^{*}}\left(\lambda^{-n}-1\right) x y & \frac{1}{2 r^{*}}\left(x^{2}+\lambda^{-n} y^{2}\right) & 0 \\
\frac{\left(\lambda^{2 n}-1\right) x}{\lambda^{n-1}\left(\lambda^{2}-1\right)} & \frac{\left(\lambda^{2 n}-1\right) y}{\lambda^{n-1}\left(\lambda^{2}-1\right)} & \lambda^{n}
\end{array}\right)
$$

where $\lambda=h^{\prime}\left(z^{*}\right)$. Moreover, if $\lambda>1(<1)$ the invariant circle has a stable (unstable) manifold contained in $\mathcal{W}_{1}$, and unstable (stable) manifold contained in $\mathcal{W}_{0}$.

Proof. Given the symmetry (20), it is enough to check (22) for points of the form

$$
\left(\sqrt{2 r^{*}}, 0, z^{*}\right)
$$

Since (22) reduces to (21) when $n=1$, it is enough to verify the induction step

$$
D g\left(\sqrt{2 r^{*}}, 0, z^{*}\right) D g^{n}\left(\sqrt{2 r^{*}}, 0, z^{*}\right)=D g^{n+1}\left(\sqrt{2 r^{*}}, 0, z^{*}\right) \text {. }
$$

The vector $(0,0,1)^{t}$ is an eigenvector of $D g\left(x, y, z^{*}\right)$ with eigenvalue $\lambda$. Since this is tangent to $\mathcal{W}_{0}$, this shows that it is the stable manifold when $\lambda<1$. Similarly the vector $\left(x, y, 2 r^{*} \frac{\lambda}{1-\lambda^{2}}\right)^{t}$ is an eigenvector with eigenvalue $\lambda^{-1}$ that is tangent to $\mathcal{W}_{1}$. The final eigenvector of $D g$ is $(y,-x, 0)^{t}$ which is tangent to $\mathcal{C}$ and has eigenvalue 1. 


\section{$5.1 \quad$ Integrable case}

In general the maps $g$ and $f$ are not integrable, even though they have a saddle connection. However, for a special choice of $h$ there is an integral. This example is related to the work of Suris [26, 27] on area-preserving integrable maps, but is distinct from the threedimensional maps found in [28] that have an invariant but which do not have a rotational symmetry.

Let $m(w)=\frac{a w+b}{c w+d}$ be the Möbius transformation on $\mathbb{R} \cup\{\infty\}$ with $a d-b c=1$. A circle map conjugate to $m$ is obtained by defining $w=\tan \pi z$, giving $h$

$$
h_{m}(z)=\frac{1}{\pi} \arctan (m(\tan \pi z)) .
$$

This map can be written more explicitly as a circle map using trigonometric identities:

$$
h_{m}(z)=z+\frac{1}{\pi} \arctan \left[\frac{b-c+(b+c) \cos (2 \pi z)+(a-d) \sin (2 \pi z)}{a+d-(a-d) \cos (2 \pi z)+(b+c) \sin (2 \pi z)}\right]
$$

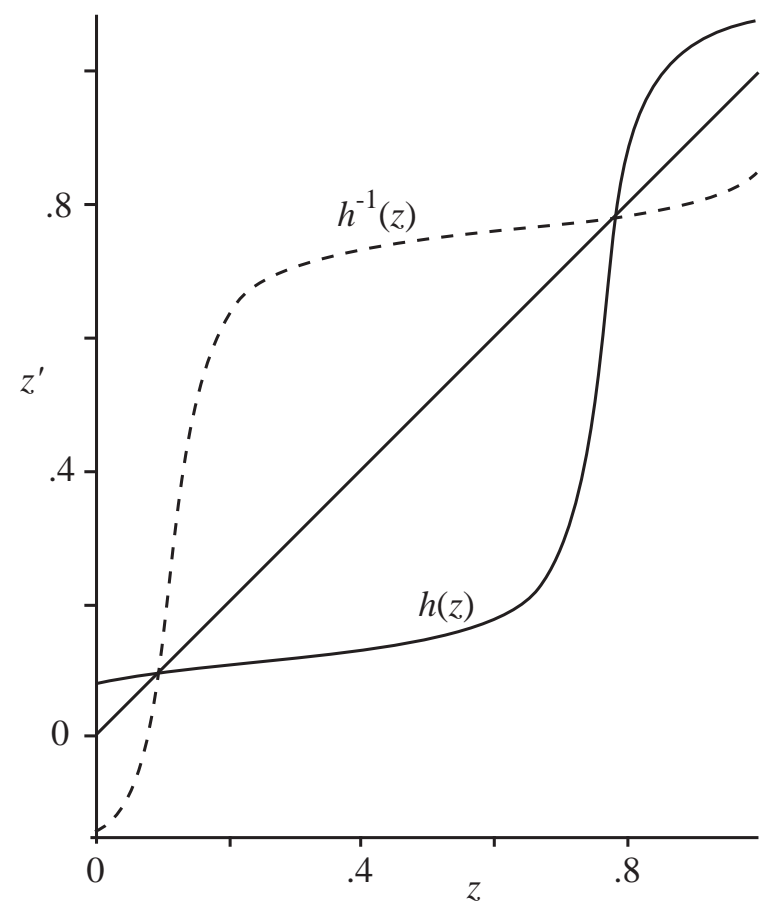

Figure 6: The circle map $h_{m}(z)$ and its inverse for $(a, b, c, d)=\left(1, \frac{1}{2}, 2,2\right)$.

Requiring $a d-b c=1$, some useful properties of this family of circle maps follow easily from its conjugacy to the Möbius transformation

- $h_{m}^{t}=h_{m^{t}}$, for all $t \in \mathbb{Z}$;

- If $|\operatorname{tr}(m)|>2$ then $h_{m}$ has two fixed points $z_{ \pm}^{*} \in[0,1)$. 
- The fixed points have multipliers $D h\left(z_{ \pm}^{*}\right)=\frac{4}{\left(\sigma \pm \sqrt{\sigma^{2}-4}\right)^{2}}>0$, where $\sigma=a+d$.

- Thus $z_{-}^{*}$ is unstable and $z_{+}^{*}$ is stable.

For any $h_{m}$, the resulting map $G$ is integrable. To see this, we show how this map is related to the Suris example. First note that $G$ can be rewritten as a second difference equation

$$
z_{t+1}-2 z_{t}+z_{t-1}=h\left(z_{t}\right)+h^{-1}\left(z_{t}\right)-2 z_{t}=F\left(z_{t}\right),
$$

where $r_{t}=z_{t+1}-h\left(z_{t}\right)+r^{*}$. Suris showed that this family is integrable when $F$ is given by

$$
F(z)=\frac{1}{\pi} \arctan \left[\frac{A \sin (2 \pi x)+B \cos (2 \pi x)+C \sin (4 \pi x)+D \cos (4 \pi x)}{1-E-A \cos (2 \pi x)+B \sin (2 \pi x)-C \cos (4 \pi x)+D \sin (4 \pi x)}\right],
$$

for any values of the parameters $A, B, C, D, E$. After some algebra one can see that our map has this form with $A=b^{2}-c^{2}, B=(a-d)(c-b), C=\frac{1}{2}(b+c)^{2}-\frac{1}{2}(a-d)^{2}$, $D=(d-a)(b+c), E=\frac{1}{2}\left(a^{2}+b^{2}+c^{2}+d^{2}\right)$.

The map $G$ with this $h$ has the integral

$$
\begin{aligned}
J\left(z, z^{\prime}\right)= & (1-E) \cos \left(2 \pi\left(z-z^{\prime}\right)\right)-A\left(\cos (2 \pi z)+\cos \left(2 \pi z^{\prime}\right)\right)+ \\
& B\left(\sin (2 \pi z)+\sin \left(2 \pi z^{\prime}\right)\right)-C \cos \left(2 \pi\left(z+z^{\prime}\right)\right)+D \sin \left(2 \pi\left(z+z^{\prime}\right)\right) .
\end{aligned}
$$

For the examples, we will use the map (19) with $h_{m}$ given by (24) with

$$
m(w)=\frac{(\nu+1) w+\nu-1}{(\nu-1) w+\nu+1}
$$

This corresponds to setting $m$ to the hyperbolic rotation matrix $a=d=\cosh (\ln (\nu))$, $b=c=\sinh (\ln (\nu))$. In this case $m^{t}$ is given by replacing $\nu$ with $\nu^{t}$; thus iteration of $h$ is extremely easy. This was also the example used in [22].

This gives a family of three-dimensional maps, $f$, with parameters $\nu$ and $\tau$. Setting $r^{*}=1$, there are invariant circles at $\left(r^{*}, z^{*}\right)=\left(1, \pm \frac{1}{4}\right)$. For this case the invariant has the form

$$
J(x, y, z)=2 \nu \cos (2 \pi r)+\left(1-\nu^{2}\right) \cos (2 \pi z) \sin (2 \pi r) .
$$

The level sets corresponding to $J=2 \nu$ give the invariant manifolds $\mathcal{W}_{0}, \mathcal{W}_{1}$, and the circles $\mathcal{C}\left(z^{*}\right)$. The level sets of $J$ are shown in Fig. 7 for the case $\nu=0.3$. Though the level sets of $J$ make it appear that $\left(\frac{1}{2}, \pm \frac{1}{4}\right)$ are also invariant; $G\left(\frac{1}{2}, \frac{1}{4}\right)=\left(\frac{1}{2},-\frac{1}{4}\right)$, so these points move downward. Moreover the curve $\left\{r=\frac{1}{2}\right\}$ has image $\left\{r=h_{\nu}^{-1}(z)-h_{\nu}(z)+\frac{1}{2}\right\}$, and this latter curve has image again of $r=\frac{1}{2}$.

\subsection{Perturbed Map}

We break the invariant surfaces by choosing a perturbation of the form (2). For the first example, we choose a composition of two simple perturbations:

$$
\begin{aligned}
& P_{1}(x, y, z)=\left(\left(1+y^{2}\right)\left(z^{* 2}-z^{2}\right), 0,0\right), \\
& P_{2}(x, y, z)=\left(0, x\left(z^{* 2}-z^{2}\right), 0\right) .
\end{aligned}
$$




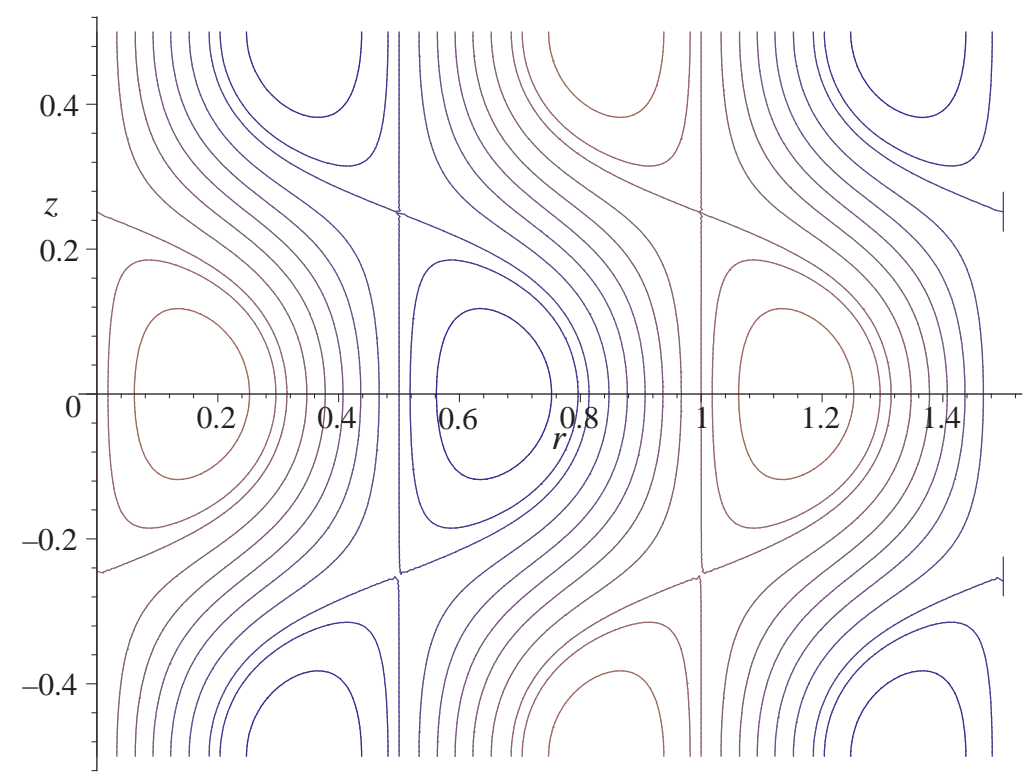

Figure 7: Levels sets of $J$ for $\nu=0.3$ Here the maximum of $J$ occurs at $z=0$ near $r=1.1$, while a minimum occurs near $r=0.7$.

Each of these maps has a nilpotent Jacobian, which implies that the maps $i d+\varepsilon P_{i}$ are volume-preserving for all $\varepsilon$. The complete perturbation is then defined as

$$
i d+\varepsilon P_{\varepsilon}=\left(i d+\varepsilon P_{2}\right) \circ\left(i d+\varepsilon P_{1}\right) .
$$

Substituting the perturbation into the computation (1) for the vector field $X_{\varepsilon}$ gives

$$
X_{0}=P_{2}+P_{1} .
$$

Fundamental domains $\mathcal{P}_{i}$ on $\mathcal{W}_{i}$ are given by the annuli bounded by the circles $\gamma_{i}=$ $\{z=0\} \cap \mathcal{W}_{i}$ and their images, $f\left(\gamma_{0}\right)=\{z=h(0)\} \cap \mathcal{W}_{0}$ or $f\left(\gamma_{1}\right)=\left\{z=h^{-1}(0)\right\} \cap \mathcal{W}_{1}$, respectively. These can be projected onto $(z, \theta)$ coordinates for visualization. Calculation of the Melnikov sum (12) is straightforward using the adapted form $d J$ associated with the invariant (26).

We show several representative contour plots of $M_{d J}$ in Fig. 8. In the figure, positive values of $M_{d J}$ are shown as dashed lines and negative as dotted lines, while the zero contour is the solid line. For example, in the bottom-left panel $(\nu=0.275$ and $\tau=0.325)$ there are two zero contours, corresponding to the unstable and stable manifolds crossing with opposite signatures (since the algebraic flux through the fundamental annulus is zero, the zero contours must come in pairs). Since the unperturbed map takes the circle $\gamma_{1}$ to the circle $f\left(\gamma_{1}\right)$ shifting each point by $\tau$, the lower boundary of $\mathcal{P}$ can be identified with the upper boundary after shifting the latter to the right by $\tau$ (we show this shift by the arrows in Fig. 8). After this identification the fundamental annulus becomes a torus, and the zero contours correspond to a pair of circles that wrap once vertically. Thus these contours have homology type $(1,0)$. The bottom right panel also has this homology type, though the curves are very close to a bifurcation. 

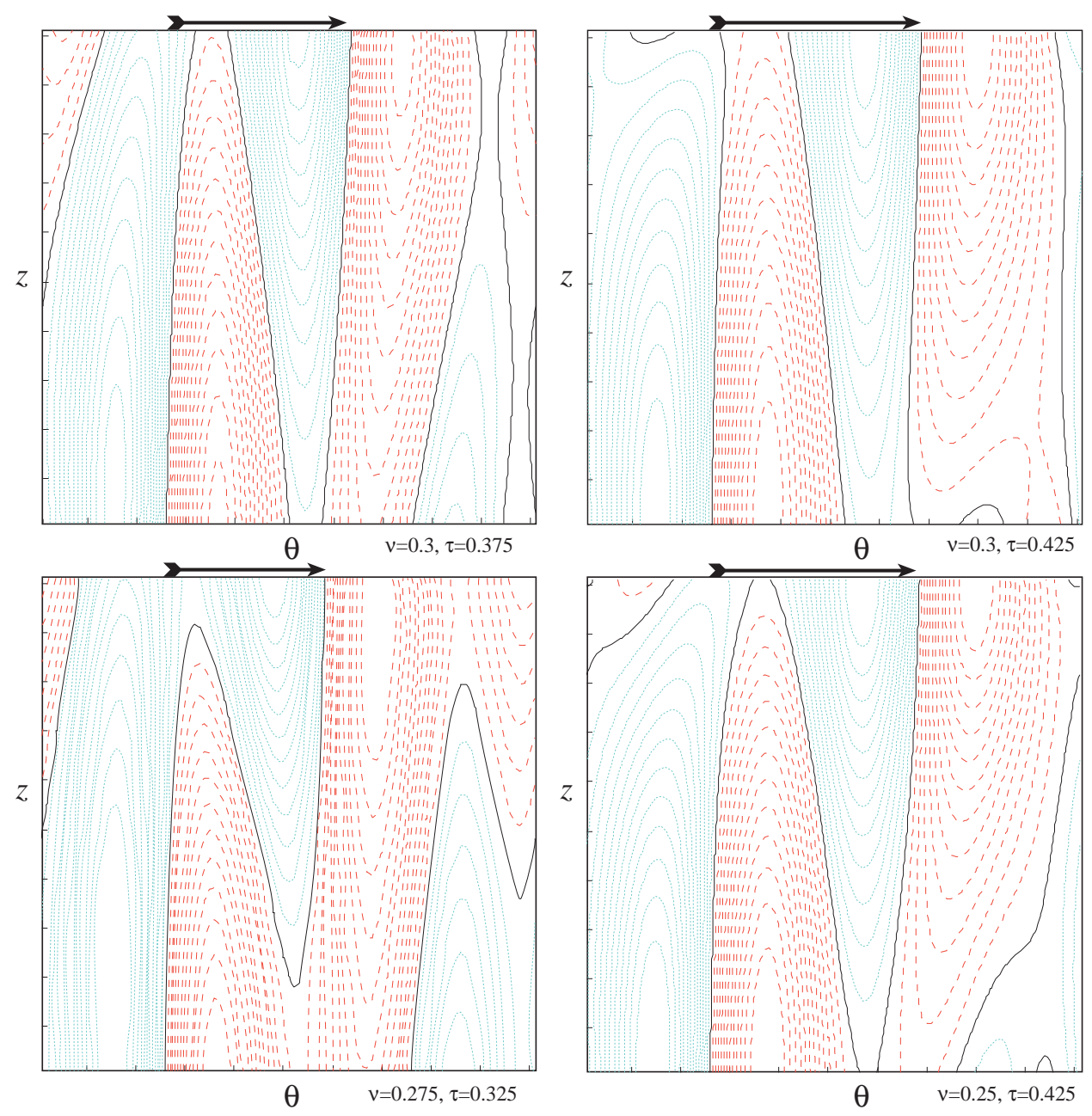

Figure 8: Contours of the Melnikov function for $\mathcal{W}_{0}$ with the perturbation (27). Shown are four values of the parameters $(\nu, \tau)$. Bounds for the figures are $z=\left[0, h^{-1}(0)\right]$ and $\theta=[0,2 \pi]$. The arrow at the top of each panel shows the translation by $\tau$.

There are several such bifurcations in homology type of the zero contours as we vary the parameters. For example in the upper left panel, the homology type is $(3,1)$ - as each zero contour moves from the bottom to the top of $\mathcal{P}$, it lags the maps translation of $\theta$ by a full circuit in three vertical transits. In the top-right panel there are two zero contours with the homology $(2,1)$. To elucidate these changes in homology, we show a bifurcation diagram in the space of the parameters in Fig. 9 . We have only found the three homology classes already mentioned.

Also shown in Fig. 9 are contours of the geometric flux

$$
\text { Flux }=\frac{1}{2} \int_{\mathcal{P}}|\Phi|
$$

as a function of $\nu$ and $\tau$. The flux is largest when $\nu$ and $\tau$ are both small, and it appears 
to get extremely small as $\nu$ approaches one. Note that there is a "valley" in the flux contours near both homology bifurcations.

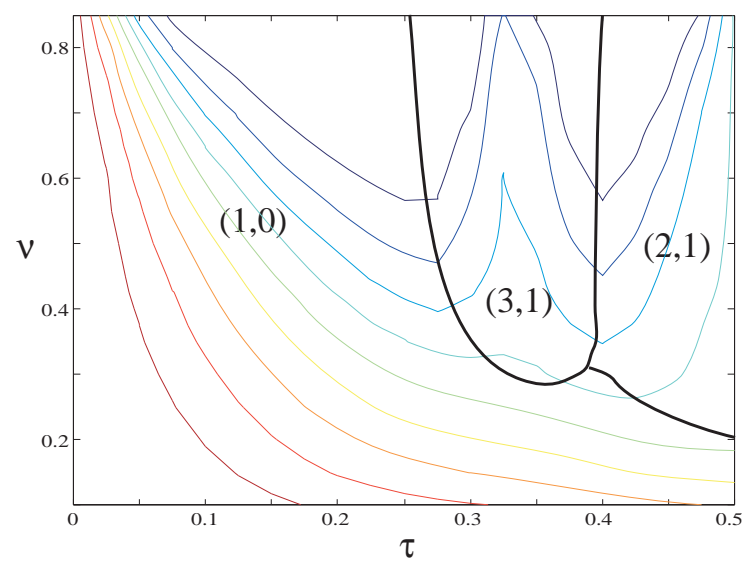

Figure 9: Contours of the geometric flux through $\mathcal{W}_{0}$ as a function of $\nu$ and $\tau$ for the perturbation (27). The nine contours are at equally spaced levels ranging from a flux of 0.09 at the lower left to 0.01 at the top. Also shown are bifurcation curves corresponding to the change in homology types of the zero contours of $M_{d J}$.

Finally, we have also studied the perturbation

$$
\begin{aligned}
& P_{1}(x, y, z)=\left(\left(1+y^{2}\right)\left(z^{* 2}-z^{2}\right), 0,0\right) \\
& P_{2}(x, y, z)=\left(0, x^{2}\left(z^{* 2}-z^{2}\right), 0\right) \\
& P_{3}(x, y, z)=\left(0,0, r-r^{*}\right)
\end{aligned}
$$

giving a perturbation vector field $X_{0}=P_{1}+P_{2}+P_{3}$. We show the bifurcation diagram for the zero contours of $M_{d J}$ for $\mathcal{W}_{0}$ in Fig. 10. For this case there appear to be only two homology types, $(1,0)$ and $(3,1)$. Again there is a "valley" in the flux near the bifurcation curve.

We have also computed the Melnikov function for the second invariant set, $\mathcal{W}_{1}$, but do not show the curves since they are very similar to those for $\mathcal{W}_{0}$.

\section{Conclusion}

We have shown that the flux-form $\Phi$ is the unique $(n-1)$-form on a codimension-one saddle connection that describes the lowest order splitting of the manifolds upon perturbation. The integral of the one-way flux over a fundamental domain characterizes the transport across the manifolds in the perturbed system. For our example, the magnitude of the flux is strongly correlated with bifurcations in the homology of the crossing curves - near a bifurcation the flux is small. It would be nice to understand if this is a general feature of transport for volume-preserving maps. 


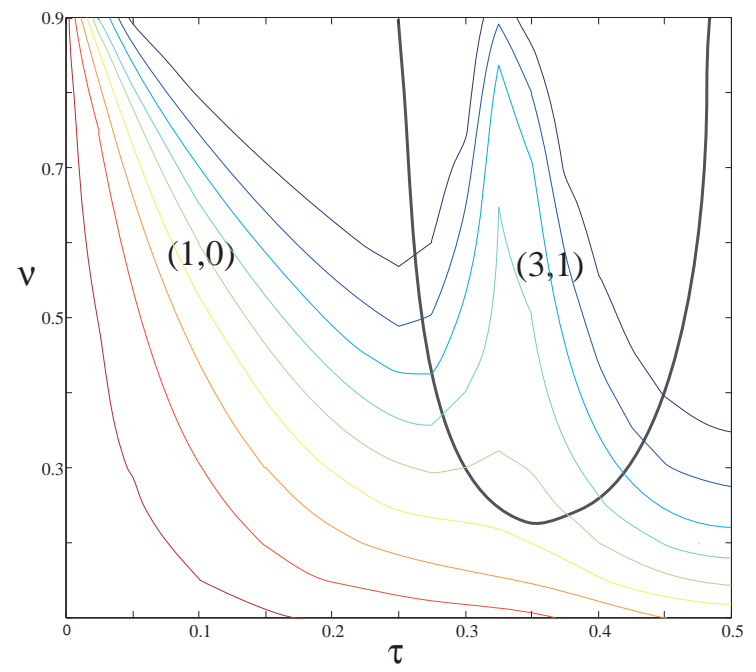

Figure 10: Contours of the geometric flux through $\mathcal{W}_{0}$ as a function of $\nu$ and $\tau$ for the perturbation (28). Also shown are bifurcation curves corresponding to the change in homology types of the zero contours of $M_{d J}$. In this case there is only one curve of bifurcation, corresponding to $(1,0) \longleftrightarrow(3,1)$.

In the future we also hope to study the evolution of the full manifolds numerically, to compare with our Melnikov results. We would also like to develop a nonperturbative method to compute the geometric flux, analogous to the action techniques for symplectic maps [10]. With this, we would like to verify that the geometric flux quantifies the transport observed numerically.

\section{References}

[1] P. Holmes. Some remarks on chaotic particle paths in time-periodic, threedimensional swirling flows. Contemp. Math, 28:393-404, 1984.

[2] Y. T. Lau and J. M. Finn. Dynamics of a three-dimensional incompressible flow with stagnation points. Physica D, 57:283-310, 1992.

[3] I. Mezic and F. Sotiropoulos. Ergodic theory and experimental visualization of invariant sets in chaotically advected flows. Physics of Fluids, 14(7):2235-2243, 2002.

[4] T. Shinbrot, M.M. Alvarez, J.M. Zale, and F.J. Muzzio. Attraction of minute particles to invariant regions of volume preserving flows by transients. Physical Review Letters, 86(7):1207-1210, 2001.

[5] Arnold V. I. and Khesin B. A. Topological methods in Hydrodynamics. SpringerVerlag, 1998.

[6] O Piro and M. Feingold. Diffusion in three-dimensional liouvillian maps. Physical Review Letters, 61:1799, 1988. 
[7] M. Feingold, L.P. Kadanoff, and O. Piro. Transport of passive scalars: KAM surface and diffusion in three-dimensional Liouvillian maps. In I. Tirapegui and D. Villarroel, editors, Instabilities and Nonequilbrium Structures II, pages 37-51. Kluwer Academic, 1989.

[8] R. S. MacKay. Transport in 3D volume-preserving flows. J. Nonlin. Sci, 4:329-354, 1994.

[9] R. S. MacKay, J. D. Meiss, and I. C. Percival. Transport in Hamiltonian systems. Physica D, 13:55-81, 1984.

[10] J. D. Meiss. Symplectic maps, variational principles, and transport. Reviews of Modern Physics, 64(3):795-848, 1992.

[11] R. S. MacKay, J. D. Meiss, and I. C. Percival. Resonances in area-preserving maps. Physica D, 27:1-20, 1987.

[12] R.W. Easton. Transport through chaos. Nonlinearity, 4:583-590, 1991.

[13] R.W. Easton, J.D. Meiss, and S. Carver. Exit times and transport for symplectic twist maps. Chaos, 3(2):153-165, 1993.

[14] V. Rom-Kedar and S. Wiggins. Transport in two-dimensional maps. Arc. Rational Mech. Anal., 109(3):239-298, 1988.

[15] R.S. MacKay and J.D. Meiss. Relationship between quantum and classical thresholds for multiphoton ionization of excited atoms. Phys. Rev. A, 37(12):4702-4706, 1988.

[16] T.J. Kaper and S. Wiggins. Lobe area in adiabatic Hamiltonian systems. Physica $D, 51: 205-212,1991$.

[17] R.W. Easton. Computing the dependence on a parameter of a family of unstable manifolds: Generalized Melnikov formulas. Non. Anal. Th. Meth. and Appl., 8:1, 1984.

[18] A. Delshams and R. Ramírez-Ros. Poincaré-Melnikov-Arnold method for analytic planar maps. Nonlinearity, 9(1):1-26, 1996.

[19] A. Delshams and R. Ramírez-Ros. Melnikov potential for exact symplectic maps. Comm. Math. Phys., 190(1), 1997.

[20] H. E. Lomelí. Applications of the Melnikov method to twist maps in higher dimensions using the variational approach. Ergod. Th. and Dyn. Sys., 17:445-462, 1997.

[21] I. Mezic and S. Wiggins. On the integrability and perturbation of three-dimensional fluid flows with symmetry. J. Nonlinear Sci., 4:157-194, 1994. 
[22] H. E. Lomelí and J. D. Meiss. Heteroclinic primary intersections and codimension one Melnikov method for volume-preserving maps. Chaos, 10(1):109-121, 2000.

[23] H. E. Lomelí. Saddle connections and heteroclinic orbits for standard maps. Nonlinearity, 9:649-668, 1996.

[24] R. Abraham and J.E. Marsden. Foundations of Mechanics. Benjamin, Reading, 1978.

[25] S. Wiggins. Global Bifurcations and Chaos: Analytical Methods, volume 73 of Applied Mathematical Sciences. Springer-Verlag, New York, 1988.

[26] Y. B. Suris. Integrable mappings of the standard type. Functional Analysis and Applications, 23:74-76, 1989.

[27] Y. B. Suris. A discrete-time Garnier system. Physics letters A, 189:281-289, 1994.

[28] A. Gomez and J.D. Meiss. Volume-preserving maps with an invariant. Chaos, 12:289$299,2002$. 